\title{
Methods for Heart Rate Variability Analysis During Sleep
}

\author{
Anna M. Bianchi, Member, IEEE, Martin O. Mendez,
}

\begin{abstract}
In the last years we have witnessed the growing interest in the heart rate variability (HRV) signal analysis during sleep. The study of the autonomic regulation during sleep allowed developing methods for automatic detection and classification of some sleep characteristics, both in physiological and pathological conditions. The main problems which require to be faced are the presence of frequent non-stationarities in the signal and the need of dealing with long term analysis, in order to provide reliable indices able to describe the whole night of sleep. In the present paper we are presenting some of the methodologies we recently employed in the study of the heart rate variability during sleep, ranging from timefrequency analysis to long time correlation. Some results are also presented, related to different applications, dealing with both physiological and pathological conditions.
\end{abstract}

Keywords: sleep, heart rate variability, respiration, autonomic control

\section{INTRODUCTION}

Historically, sleep is considered a phenomenon related to the central nervous system. From the pioneering research of Hans Berger sleep EEG was clearly described [1] and in 1968 Rechtschaffen, and Kales provided clinical guidelines for sleep classification in different stages mainly based on EEG characteristics. Sleep, in fact, is not a stable and uniform condition: during the night, 4 to 6 cycles can be recognized, during which the subject switches between nonREM to REM (Rapid Eye Movement) sleep with a periodicity of about $90 \mathrm{~min}$ [2]; further, the nonREM sleep presents different characteristics of deepness that are usually classified in three/four stages that constitute the "macrostructure". Inside each sleep stage other phasic events ("microstructure" of the sleep) may occur such as microarousals, k-complexes, CAP (Cyclic Alternating Pattern) sequences, with alternation of $\mathrm{A}$ (A1, a2 and A3, corresponding to activations) and $\mathrm{B}$ (restoring to background conditions) phases, movements and apneas [3]. In the recent years the interest has grown about involvement of other systems and organs and dedicated researches have put into evidence how sleep can be considered a "multivariate and multiorgan" phenomenon. In particular, emphasis is given to the fact that during different sleep stages and conditions, in both physiological and pathological situations, Autonomic Nervous System (ANS) behavior changes, influencing a multiplicity of organs: not only cardiovascular system, but also respiratory, muscular, endocrine-metabolic systems and

Manuscript received February, $4^{\text {th }}, 2013$

A. M. Bianchi is with Politecnico di Milano, Dept. Of Electronic, Information and Biomedical Engineering, P.zza Leonardo da Vinci 32, Milan, Italy (tel: +390223993342, email: annamaria.bianchi@polimi.it).

M. O. Mendez is with Facultad de Ciencias, Diagonal Sur S/N, Zona Universitaria, San Luis Potosi, S.L.P., Mexico (e-mail: mmendez@galia.fc.uaslp.mx). others [4]. Therefore direct connections among central, peripheral and autonomic nervous systems are most often found. Hence, a multivariate analysis on more signals including EEG, HRV, respiration, EMG and others derived from different organs, can help in better understanding the sleep mechanisms and the connections among different physiological systems [4 -6]. In the last years great emphasis was given to the study of the HRV during sleep, as a tool for the analysis of the ANS, and the obtained parameters have been used not only for a better understanding of the sleep physiology, but also for automatic classification of sleep stages and detection of sleep disturbances. This gave rise to the study and development of devices for home sleep monitoring, wearable or integrated into the living ambient, with many applications to different pathologies [7]. In this paper we provide an overview of the methodologies we used for HRV analysis and of the main parameters and features employed at this purposes, both in physiological and pathological conditions.

\section{METHODS}

\section{A. Spectral and Cross-Spectral analysis}

The frequency analysis of the HRV signal provides a view of the status of the ANS: in fact the power distribution and the central frequency of the spectral components vary in relation to changes in autonomic modulation of the HR and depending on the central nervous system state [8]. The autonomic regulation is modulated in relation to the different sleep stages and this modulation can be quantified through spectral parameters obtained through the frequency analysis of the HRV signal. In addition, the extension of the frequency analysis to bivariate formulation allows achieving information about the coupling with other physiological parameters, for example between HRV and respiratory activity [9]. This provides parameters related to the cardiopulmonary coupling and find an interesting field of application in sleep breathing disorders studies, such as nocturnal apneas and athsma.

\section{B. Time-frequency analysis}

The traditional spectral analysis, described in the previous section, requires stationarity of the signals on time windows of some minutes (3-5 min corresponding to 200-300 heart beats), thus presents some drawbacks when applied to sleep studies. In fact, sleep is not a uniform condition, but is characterized by transitions between stages, movements, micro-arousals, apneas, etc., that are reflected on the HR. In addition, the clinical sleep staging is based on time frames of $30 \mathrm{sec}$. that are too short for a reliable HRV frequency analysis. For those reasons, time-frequency analysis has been proposed, which can be performed through different approaches: adaptive AR models [10, 11], time-frequency distributions (Wigner - Ville, WV) [12], wavelet transform 
(WT), Empirical Mode Decomposition (EMD) [13]. All the above mentioned methodologies allow the estimation of the spectral parameters on very short time frames (up to single heart beat), and are then useful for the analysis of the ANS balance during transient phenomena such as microarousals and apneas [14], or for sleep staging on time windows of 30s.

\section{Time domain analysis}

Phasic events on the EEG during sleep affect the HRV signal that usually presents a characteristic tachycardia episode. In particular, during A phases of CAP sleep we can observe RR interval shortening which intensity may vary according to the A phase type (A1, A2 or A3). Such episodes are usually interpreted as sympathetic activation, however, their time duration (in the range of a few seconds) does not allow a reliable frequency analysis. For this reason they are usually analyzed in the time domain in terms of latency and amplitude in respect of the A phase onset on the EEG [4].

\section{Long term correlation analysis}

In sleep study the interest is also on the night considered as a whole, and the information coming from the HRV analysis should be summarized in a few indices. In the last decade many different parameters have been proposed for the estimation of the long term correlation characteristics in HRV time series. Some of them are briefly described in the following:

Sample Entropy (SampEn)

$\operatorname{SampEn}(\mathrm{m}, \mathrm{r})$ measures, with a tolerance $r$, the regularity of patterns comparing them to a given pattern of length $\mathrm{m}(\mathrm{m}$ and $r$ are fixed values: $m$ is the detail level at which the signal is analyzed and $r$ is a threshold, which filters out irregularities) $[15,16]$. The adopted parameters in the present application are $\mathrm{m}=2$ and $\mathrm{r}=0.2$.

Multiscale Entropy (MSE)

MSE was proposed in order to capture HRV fluctuations at different degrees of resolution, i.e. in a multiscale manner [17]. The first step to compute MSE is the construction of the coarse-grained time series. Given a time series of $\mathrm{N}$ points $\{\mathrm{xi}\}$, the coarse-grained time series $\{\mathrm{y}(\tau)\}$, determined by the factor $\tau$, are constructed as follows:

$$
\mathbf{y}_{j}^{(\tau)}=\frac{1}{\tau} \sum_{i=(j-1) \tau+1}^{j \tau} x_{i}, \text { for } 1 \leq j \leq N-\tau
$$

For each of these new time series, an entropy measure is calculated and the obtained value is plotted as a function of the coarse-graining scale factor. In this work the proposed indices were the average of the MSE values estimated over the time scale from 1 to 5 (MSE1) and from 10 to 20 (MSE2).

\section{Lempel-Ziv Complexity (LZC)}

The measure of complexity introduced by Lempel and Ziv assesses the so-called algorithmic complexity, which is defined according to information theory as the minimum quantity of information needed to define a binary string. In case of random strings, the algorithmic complexity is the length of the string itself. In fact any compression effort will produce an information loss. The LZC quantifies the rate of new patterns arising with the temporal evolution of the signal. In order to estimate the LZC for a biological signal, it is necessary to transform the time series into symbolic sequences. The algorithm to assess LZC and the coding procedure is fully described in [15]. In this work we adopted both the binary $\mathrm{LZC}(2)$ and the ternary $\mathrm{LZC}(3)$ coding procedure.

\section{Detrended Fluctuation Analysis (DFA)}

The DFA can be simply defined as a modified root mean square analysis of a random walk [18]. Briefly, the time series to be analyzed is firstly integrated. Next, the integrated time series is divided into boxes of equal length, $\mathrm{n}$. In each box of length $\mathrm{n}$, a least squares line which fits the data (representing the trend in that box) is estimated. Next, the integrated time series is detrended by subtracting the local trend in each box. The root-mean-square fluctuation $\mathrm{F}(\mathrm{n})$ of this integrated and detrended time series is calculated. This computation is repeated over all time scales (box sizes) to characterize the relationship between $\mathrm{F}(\mathrm{n})$, the average fluctuation, and the box size $n$. A linear relationship on a log-log plot indicates the presence of power law (fractal) scaling. Under such conditions, the fluctuations can be characterized by a scaling exponent $v$, the slope of the line relating $\log F(n)$ to $\log n$. In this work two scaling exponents are proposed: one represents an estimation of the short-term fluctuations $(\mathrm{a} 1(\mathrm{n}=4-16))$ and one of the longterm fluctuations (a2) $(\mathrm{n}=16-64)$.

\section{1/f Slope}

The slope of the power-low regression line of HRV fitted to the power spectrum for $\mathrm{f}<0.01 \mathrm{~Hz}$ [19]. This index is strongly correlated to the DFA indices when a scaling law is present, but it does not permit to separately analyze the short- and long-term components, as for the DFA.

\section{RESUlts}

\section{A. Spectral and Cross-Spectral analysis}

In [9] polysomnographic sleep recordings were performed in 11 healthy women and the HRV signal and the respiration signal were obtained. The spectral and crossspectral parameters of the HRV signal and of the respiration signal were evaluated in order to estimate the autonomic regulation and the cardiopulmonary coupling related to the different sleep stages. Results confirmed previous findings present in literature: a sympatho-vagal balance shift toward parasympathetic modulation during NREM sleep and toward sympathetic modulation during REM sleep. Further, spectral analysis of the HRV signal and of the respiration signal indicated an higher respiration regularity during deep sleep, and a higher coherence between the HRV and the respiration signals at the respiratory frequency during NREM sleep. Fig.1 shows an example of the analysis: the left column is related to HRV frequency analysis, the central column is related to respiration, while the right column shows the squared coherence function between the two signals; from top to the bottom wake and the different sleep stages are represented.

\section{B. Time frequency analysis}

Fig. 2 shows a typical example of the time-variant, beat-tobeat analysis of the HRV signal recorded during night. Each 


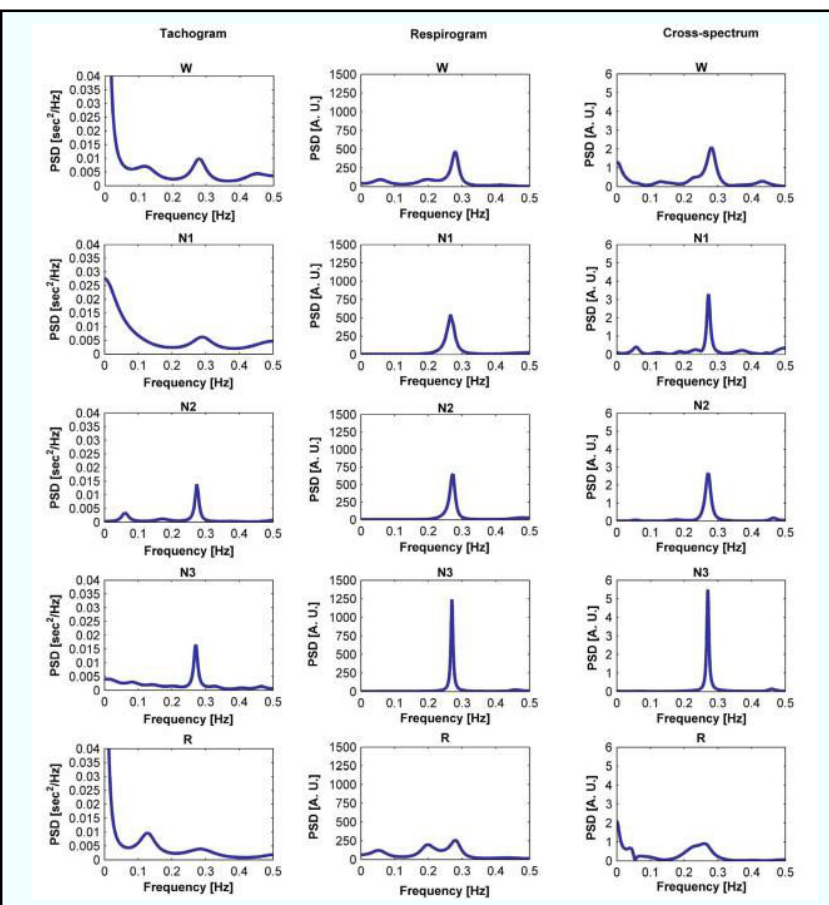

Figure 1. Power spectral density (PSD) computed from the tachogram of a subject during wakefulness (W), sleep stages N1, N2, and N3 and REM sleep (Left column); PSD computed from the respirogram of the same subject during the same periods, (Central column); crossspectrum between the tachogram and the respirogram of the same subject during the same periods (right panel) [8].

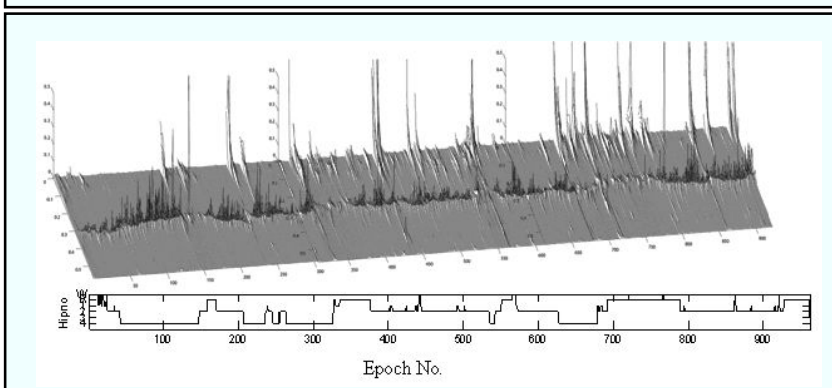

Figure 2. Example of time frequency analysis during a whole night of sleep is shown in top panel, while the bottom panel shows the hypnogram obtained through a standard polysomnography

spectrum corresponds to a single $\mathrm{RR}$ value, and all the spectra are represented as a function of time in the compressed spectral array form (CSA). Below the CSA representation there is the hypnogram, obtained from the standard polysomnography through the vusula analysis of EEG, EMG and EOG. The spectral components are clearly modulated by the different sleep stages. This suggested using spectral parameters coming from HRV for sleep staging [10]. The same analysis allowed also obtaining features for the detection of sleep apneas [14]. Other time frequency methods, such as WV Distribution, revealed to be effective in the study of the autonomic activations during arousals [12], while WT and EMD allowed the detection of sleep apneas [13]. Starting from the time frequency distributions, many different features can be extracted, also on very short time frames that can be fed into proper classification algorithm for an automatic detection and classification of events of interest.

\section{Time domain analysis}

One of the interests in the CAP sleep study is to assess the effect of A phases on the autonomic nervous system. It was demonstrated that A2 and A3 clearly modify the HRV. However, A1 influence is controversial. We analyzed A1 phases on the HRV evaluating amplitude and latency of the minimum RR value in the 25 seconds after the A1 onset on the EEG and compared these indexes by taking randomly segments of 25 seconds during the NREM sleep. As it can be observed in Fig.3, the amplitude reduction of the HRV with and without A1 activation are similar in $25 \mathrm{sec}$, however, the time where the minimum RR occurs shows differences. HR reduction with A1 influence presents a well located peak around 5 seconds, while HR without A1 activation presents a uniform distribution. This means, that A1 activations really have an influence on the HRV which is not casual even if not easily observable.

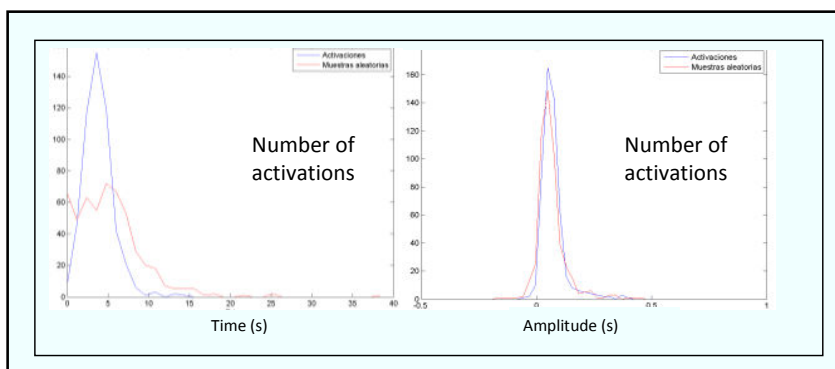

Fig. 3. Left panel: Distribution of occurrence time of minumum HR for A1 activations (blue) and random sequences (red). Right panel: Distribution of amplitude changes of the minimum HR for A1 activations (blue) and random sequences (red).

\section{E. Long term correlation analysis}

TABle I. LONG TERM CORRELATION PARAMETERS

\begin{tabular}{|c|c|c|c|c|c|}
\hline & \multicolumn{2}{|c|}{ Healthy } & \multicolumn{3}{|c|}{ Unhealthy } \\
\hline & HSE & $L S E$ & Insomnia & Apnea & $\begin{array}{l}\text { Heart } \\
\text { failure }\end{array}$ \\
\hline $\begin{array}{c}\text { SampEn } \\
(2,0.2)\end{array}$ & $1.20 \pm 0.07$ & $1.19 \pm 0.09$ & $0.91 \pm 0.12 \dagger$ & $\begin{array}{c}0.97 \pm 0.04 \\
\dagger\end{array}$ & $0.90 \pm 0.10$ \\
\hline MSE1 & $0.88 \pm 0.19$ & $0.82 \pm 0.14$ & $0.75 \pm 0.17$ & $0.96 \pm 0.28$ & $\begin{array}{c}0.65 \pm 0.16 \\
\dagger\end{array}$ \\
\hline MSE2 & $0.98 \pm 0.25$ & $1.02 \pm 0.17$ & $0.92 \pm 0.19$ & $\begin{array}{c}1.47 \pm 0.28 \\
\dagger\end{array}$ & $0.95 \pm 0.42$ \\
\hline $\operatorname{LZC}(2)$ & $0.92 \pm 0.06$ & $0.92 \pm 0.03$ & $0.92 \pm 0.04$ & $0.93 \pm 0.04$ & $\begin{array}{c}0.87 \pm 0.10 \\
\dagger\end{array}$ \\
\hline $\operatorname{LZC}(3)$ & $0.79 \pm 0.05$ & $0.80 \pm 0.03$ & $0.78 \pm 0.07$ & $\begin{array}{c}0.90 \pm 0.04 \\
\dagger\end{array}$ & $0.80 \pm 0.09$ \\
\hline DFA a1 & $1.18 \pm 0.22$ & $1.20 \pm 0.19$ & $1.31 \pm 0.12$ & $\begin{array}{c}1.44 \pm 0.27 \\
\dagger\end{array}$ & $\begin{array}{c}0.92 \pm 0.23 \\
\dagger\end{array}$ \\
\hline DFA a2 & $1.03 \pm 0.09$ & $1.05 \pm 0.08$ & $1.01 \pm 0.08$ & $\begin{array}{c}0.85 \pm 0.09 \\
\dagger\end{array}$ & $1.05 \pm 0.10$ \\
\hline 1/f slope & $0.71 \pm 0.19$ & $0.58 \pm 0.32$ & $0.95 \pm 0.23$ & $0.86 \pm 0.49$ & $\begin{array}{c}1.34 \pm 0.50 \\
\dagger\end{array}$ \\
\hline
\end{tabular}

HSE = high sleep efficiency (higher than 85\%), LSE = low sleep efficiency (lower than $85 \%$ ), $\dagger=$ a significant difference with respect to HSE group (p-value < 0.05) [20]

Parameters, able to measure the regularity and complexity of the HRV signal and to of time series, are calculated on several recordings acquired during the night. Different groups of subjects were analyzed: healthy subjects with high sleep efficiency (HSE), healthy subjects with low sleep 


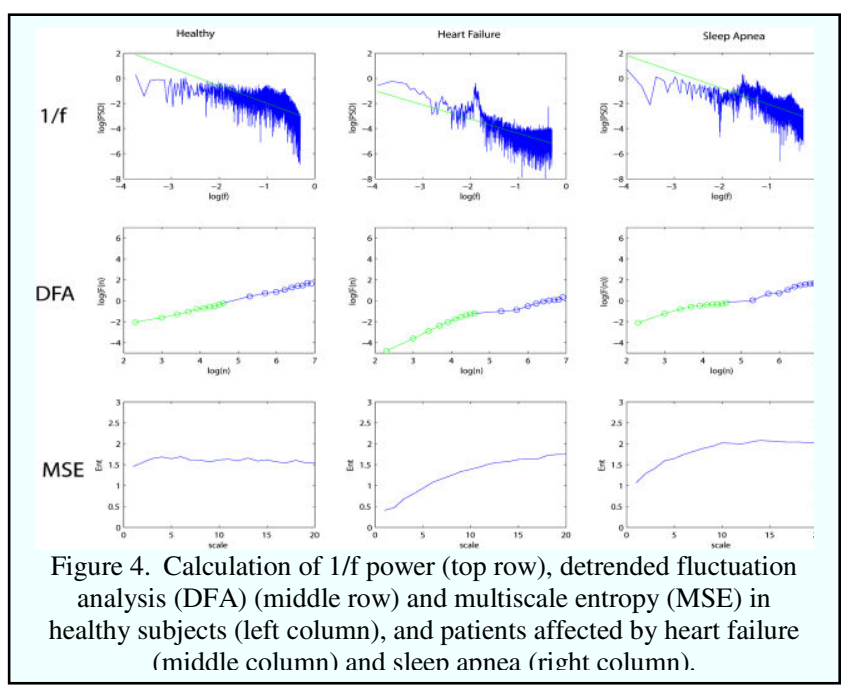

efficiency (LSE), subjects affected by insomnia (insomnia), heart failure patients (heart failure), subjects affected by obstructive sleep apnea (apnea). Some of the evaluated parameters are graphically shown in Fig.4, while their mean values with the standard deviations are reported in Tab. I. When compared with normal subjects, the pathological groups showed some significant differences. In particular heart failure patients have significant lower entropy and complexity values, whereas apnea patients show an increased irregularity when compared with normal subjects with high sleep efficiency.

\section{CONCLUSION}

In the present paper we have provided a short, and surely non exhaustive, review of some methodologies and processing techniques we have recently proposed for the analysis of the HRV signal during sleep. The described results confirm the multiorgan and multisystem involvement during sleep. In particular, the autonomic nervous system is strongly modulated, and the sympatho-vagal balance shows great variations along the night, in relation to the different sleep stages, or in correspondence of sleep events (apneas, microarousals, etc.), both physiological and pathological. It is worth remembering that HRV signal presents the advantage of reliable acquisitions, also in noisy conditions; in addition, it can be easily recorded also through wearable sensors or through sensors integrated into the bed, thus it is a good candidate for continuous monitoring of sleep quality and sleep disturbances at home [7]. This, however, requires proper processing tools. In this paper we have presented methodologies ranging from beat-to-beat analysis (then on very short periods) to whole night analysis. However, all these tools are intended to evaluate indices able to describe the whole sleep period, thus providing synthetic parameters to the physicians that could be integrated with the traditional sleep analysis for a better understanding of the underlying mechanisms.

\section{REFERENCES}

[1] Berger, H, Uber das elektrenckephalogramm des menschen, 35-, Apr. 1929

[2] Rechtschaffen, A., and Kales, A.E., A manual of standardized terminology, techniques and scoring system for sleep stages of human subjects, Brain information Services/Brain Research Institute, UCLA, 1968

[3] M. G. Terzano, L. Parrino, A. Sherieri, R. Chervin, S. Chokroverty, C. Guilleminault, M. Hirshkowitz, M. Mahowald, H. Moldofsky, A. Rosa, R. Thomas and A. Walters, "Atlas, rules, and recording techniques for the scoring of cyclic alternating pattern (CAP) in human sleep," Sleep Med., vol. 2, pp. 537-553, 2001.

[4] Bianchi AM, Ferini-Strambi L, Castronovo V, Cerutti S. Multivariate and multiorgan analysis of cardiorespiratory variability signals: the CAP sleep case. Biomed Tech (Berl). 2006;51(4):167-73.

[5] Blasi, A., Jo, J., M, Khoo, Cardiovascular Variability after Arousal from Sleep: Time-Varying spectral Analysis, J Appl Physiol, 95, 1394-1404, 2003.

[6] Sforza, E., Jouny, C., Ibanez, V. Cardiac Activation During Arousal in Humans: Further Evidence for Hierarchy in the Arousal Response, Clin Neurophysiol , 111, 1611-1619, 2002

[7] Kelly J.M., Strecker R.E., Bianchi M.T., Recent developments in home sleep-monitoring devices, ISRN Neurology, vol. 2012, pp.1-10, 2012.

[8] Task Force of the European Society of Cardiology and the North Amer- ican Society of Pacing and Elec- trophysiology. (1996). Heart rate variability. Standards of measurement, physiological interpretation and clinical use. Eur.HeartJ. 17, 354-381.

[9] Cabiddu R, Cerutti S, Viardot G,Werner S, Bianchi AM., Modulation od the sympatho-vagal balance during sleep: frequency domain study of the heart rate variability and respiration, Front Physiol. 2012;3:45 doi: 10.3389/fphys.2012.00045. Epub 2012 Mar 9

[10] M. O. Mendez, M. Matteucci, V. Castronovo, L. Ferini-Strambi, S. Cerutti, A.M. Bianchi. (2010). Sleep staging from Heart Rate Variability: time-varying spectral features and Hidden Markov Models. Int. J. of Biom. Eng. And Techn., vol. 3; p. 246-263

[11] Bianchi A.M., M. O. Mendez, S. Cerutti (2010). Processing of Signals Recorded Through Smart Devices: Sleep-Quality Assessment. IEEE Trans. on Inf. Tech in Biomedicine, vol. 14; p. 776-785,

[12] M.O. Mendez, A. M. Bianchi, N. Montano, V. Patruno, E. Gil, C. Mantaras, S. Aiol, S. Cerutti, On Arousal from Sleep: TimeFrequency Analysis. Med. \& Biol. Eng. \& Comp., 2008, vol. 46; p. 341-51.

[13] Mendez M.O, Corthout J., Van Huffel S., Matteucci M., Penzel T., Cerutti S., Bianchi A.M. (2010). Automatic screening of obstructive sleep apnea from the ECG based on empirical mode decomposition and wavelet analysis. Physiol. Measurements, vol. 31; p. 273-289,

[14] Mendez M., Bianchi A.M., Matteucci M., Cerutti S., Penzel T. (2009). Sleep Apnea Screening by Autoregressive Models from a Single ECG Lead. IEEE Trans. on Biom. Eng., vol. 12; p. 2838-2850,

[15] Richman JS, Moorman JR. Physiological Time- Series Analysis using Approximate Entropy and Sample Entropy. Am J Physiol Heart Circ Physiol 2000; 278: H2039-H2049.

[16] Pincus SM. Approximate Entropy as a Measure of System Complexity. Proc Natl Acad Sci USA 1991; 88 (6): 2297-2301.

[17] Costa M, Goldberger AL, Peng CK. Multiscale Entropy Analysis of Complex Physiologic Time Series. Physical Review Letters 2002; 89 (6): $1-4$.

[18] Ferrario M, Signorini MG, Magenes G. Comparison between Fetal Heart Rate Standard Parameters and complexity Indexes for the Identification of Severe Intrauterine Growth Restriction. Methods Inf Med 2007; 46: 186-190.

[19] Bigger JT, Steinman RC, Rolnitzky LM, Fleiss JL, Albrecht P, Cohen RJ. Power law behavior of RRinterval variability in healthy middleaged persons, patients with recent acute myocardial infarction, and patients with heart transplants. Circulation 1996; 93: 2142-2151.

[20] Bianchi A.M., Mendez M. O., Ferrario M., Ferini-Strambi L., Cerutti S. (2010). Long-term correlations and complexity analysis of the heart rate variability signal during sleep. Comparing normal and pathologic subjects. Methods of Information in Med., vol. 49; p. 479-483. 